Pacific Journal of Mathematics

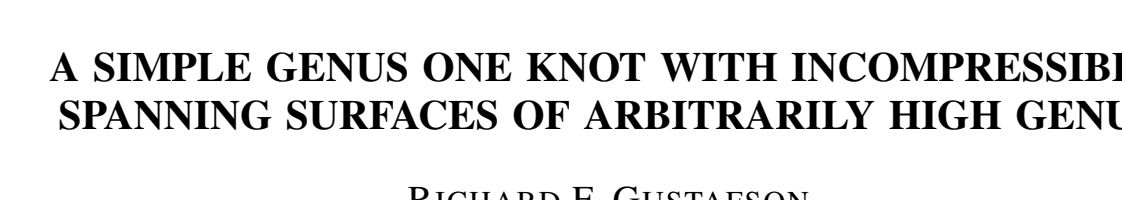




\title{
A SIMPLE GENUS ONE KNOT WITH INCOMPRESSIBLE SPANNING SURFACES OF ARBITRARILY HIGH GENUS
}

\author{
RICHARD F. GUSTAFSON
}

\begin{abstract}
The following theorem is proved. There exists a simple genus one knot with incompressible spanning surfaces of arbitrarily high genus.
\end{abstract}

1. Introduction. H. C. Lyon in [2] proved that there exists a genus one knot which has incompressible spanning surfaces of arbitrarily high genus. Lyon's knot has companions and the companions are essential to his discussion. Presented in this paper is a knot of genus one which is shown to have no companions (is simple) but which has incompressible spanning surfaces of arbitrarily high genus. The discussion is in the PL category and all knots and surfaces are tamely embedded in $S^{3}$. The notation and terminology generally follow that of [2], [3], [4], [1]. All surfaces are nonsingular unless otherwise indicated. When two surfaces are discussed it will be assumed that they are in general position so that their intersection consists of at most disjoint simple closed curves (sce) and spanning arcs.

2. The example. The knot $K$ is shown in Figure 1. Figure 2 shows a singular disc $D^{*}$ bounded by $K$. Figure 3 shows $D^{*}$ with the singularities removed by cutting out two discs $D^{\prime}, D^{\prime \prime}$ from $D^{*}$. In Figure 3 an annulus $H$ has been attached to the "hatched" side of $D^{*}$ along the two boundaries $\partial D^{\prime}, \partial D^{\prime}$. The annular tube $H$ surrounds a part of $D^{*}$. Thus Figure 3 shows an orientable surface $S(-1)$ of genus one spanned by $K$, therefore $K$ has genus at most one.

3. Some preliminaries. Ball (3-cell) $\widetilde{Q}$ is selected as in Figure 4. Sphere (2-sphere) $C=\partial \widetilde{Q}$ contains two disjoint subdises $M(1)$ and $M(4)$ which contain the points $K \cap C$ in two special classes. $\widetilde{Q}$ is subdivided into three subballs $Q(1), Q(2), Q(3)$ by two subdises $M(2)$ and $M(3)$ as illustrated in Figure 4 so that each sphere $C(i)=$ $\partial(Q(i)), i=1,2,3$, contains two $\operatorname{discs} M(i)$ and $M(i+1)$ which have the points of $K \cup C(i)$.

Figure 5 illustrates $K \cap Q(i), i=1,2,3$. There are four simple arcs $a(i, 1), a(i, 2), b(i, 1), b(i, 2)$ of $K \cap Q(i)$. Figure 6 shows how each of these arcs is completed by one of four disjoint simple arcs $\alpha(i, 1), \alpha(i, 2), \beta(i, 1), \beta(i, 2)$ in $C(i)$ so that each $b(i, j) \cup \beta(i, j), j=$ 


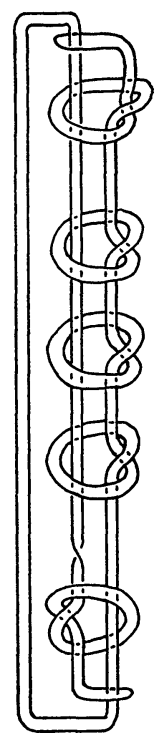

FIG. 1

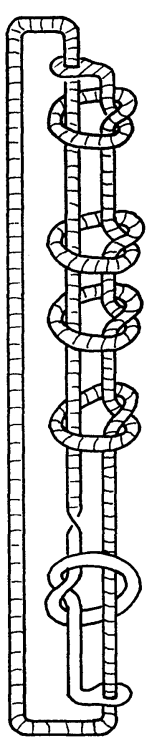

Fig. 2

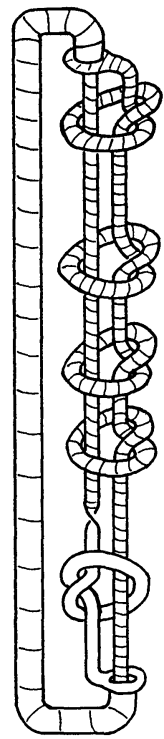

FIG. 3

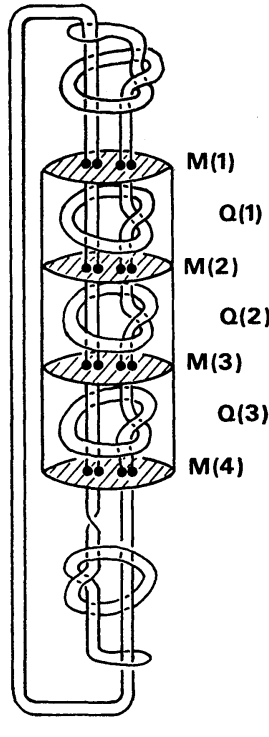

FIG. 4

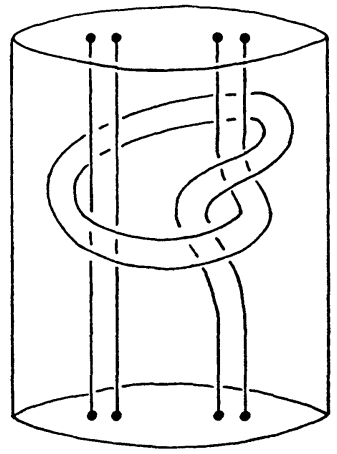

FIG. 5

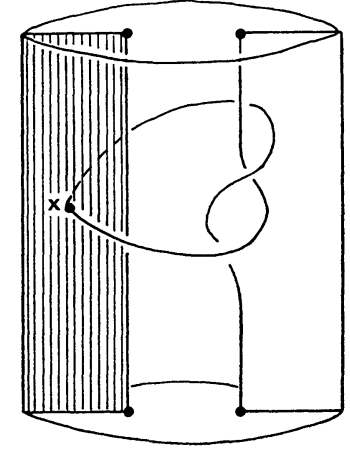

FIG. 6

1,2 , is a trefoil knot and each $a(i, j) \cup \alpha(i, j), j=1,2$, is a circle which bounds a disc in $Q(i)$ and which has linking number +1 with each trefoil knot.

Consider the case illustrated in Figure 6 for some $i \in\{1,2,3\}$, a pair $a \cup \alpha=a(i, j) \cup \alpha(i, j), b \cup \beta=b(i, j) \cup \beta(i, j)$, some $j \in\{1,2\}$. Let disc $D(i) \subseteq Q(i)$ be such that $\partial D(i)=a \cup \alpha, D(i) \cap C(i)=\alpha$, and $D(i) \cap b=\{x\}$ where $x$ is a point in the interior of $D(i)$ and in the interior of $b$.

LEMMA 3.1. Suppose disc $E \cong Q(i)-(a \cup b)$ is such that $\partial E=$ $E \cap(C(i)-(a \cup b)) . \quad E$ divides $Q(i)$ into subballs $Q^{\prime}, Q^{\prime \prime}$ such that $Q(i)=Q^{\prime} \cup Q^{\prime \prime}$ and $Q^{\prime} \cap Q^{\prime \prime}=E$. Then it must be that $a \cup b$ is contained in just one of the subballs $Q^{\prime}, Q^{\prime \prime}$. 
Proof. Suppose not. Then $a \subseteq Q^{\prime}$ and $b \subseteq Q^{\prime \prime}$, as $E \cap(a \cup b)=\varnothing$. Now $E$ separates $C(i)$ into two dises $C^{\prime}, C^{\prime \prime}$ such that $C^{\prime} \cap C^{\prime \prime}=\partial E$ and $C^{\prime} \cup E=\partial Q^{\prime}, C^{\prime \prime} \cup E=\partial Q^{\prime \prime}$. Since $\partial \alpha=\partial a \subseteq C^{\prime}-\partial C^{\prime}=C^{\prime}-\partial E$, there is no loss in generality to assume that $\alpha \subseteq C^{\prime}-\partial C^{\prime}$. Assume $E \cap D(i)$ is minimal so that $E \cap D(i)$ consists of sces each of which, $\gamma$, bounds a subdisc $D(\gamma)$ of $D(i)$ which contains $x$. Let $\gamma$ be an "innermost" such sce so that $D(\gamma)$ contains no other curves of $E \cap$ $D(i) . \gamma$ also bounds a subdisc $E(\gamma)$ of $E . \quad E(\gamma) \cap(a \cup b)=\varnothing, E(\gamma) \cap$ $D(\gamma)=\gamma$, so $E(\gamma) \cup D(\gamma)$ is a sphere in the interior of $Q(i)$, which bounds a subball of $Q(i)$, yet has just one point $x$ of the interior of $b$. This is impossible. Hence, Lemma 3.1 is proved.

\section{A corollary of Lemma 3.1 is}

LEMMA 3.2. If disc $E \cong Q(i)-K$ is such that $\partial E=E \cap(C(i)-K)$ then $\partial E$ bounds a disc in $C(i)-K$ and just one of $K \cap Q(i) \subseteq Q^{\prime}$ or $K \cap Q(i) \subseteq Q^{\prime \prime}$ holds.

The discussion continues in the context of Lemma 3.1. Two more bordered surfaces $F(i)$ and $B(i)$ are determined in $Q(i)$.

$F(i)$ is as illustrated in Figure 7. $F(i)$ is a surface of genus one which spans trefoil $b \cup \beta . \quad F(i) \cap C(i)=\beta$ and $F(i)$ is composed of two disc $F(i, 1), F(i, 2)$ connected by the three twisted rectangles shown. $a \cap F(i)=a \cap F(i, 2)$ is point $y$ and $D(i) \cap F(i, 2)$ is segment $\overline{x y}$.

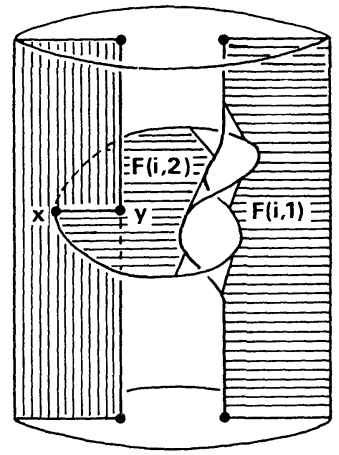

FIG. 7

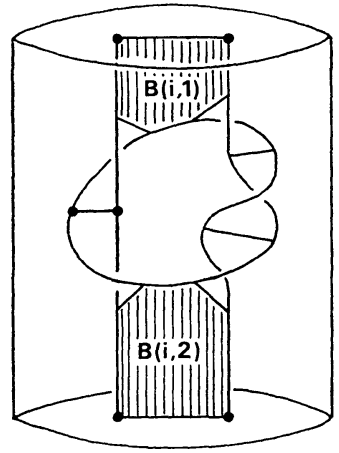

FIG. 8

Figure 8 shows Möbius band $B(i) . \quad \partial B(i)$ consists of $a, b$, an arc in $M(i)$ which connects the two points $(a \cup b) \cap M(i)$, and an arc in $M(i+1)$ which connects $(a \cup b) \cap M(i+1)$. The last two ares are $B(i) \cap C(i) . \quad B(i)$ consists of two discs, $B(i, 1)$ and $B(i, 2)$, each of which meets $C(i)$ in one arc of $B(i) \cap C(i)$, connected by the five 
twisted rectangles shown-two of these twisted rectangles share edge $\overline{x y}$.

Define $\sigma=\alpha \cup(B(i) \cap M(i)) \cup(B(i) \cap M(i+1)) \cup \beta . D(i), F(i), B(i)$ are positioned so that $D(i) \cap F(i)=\overline{x y}=D(i) \cap B(i), F(i) \cap B(i)$ consists of four line segments one of which is $\overline{x y}$ and the other three connect the double points of the projection of $b$ into a disc cut from $C(i)$ by $\sigma$. Each twisted rectangle of $F(i)$ intersects just one twisted rectangle of $B(i)$ in a segment.

(3.0) Let $A$ be an annulus which is properly embedded in $Q(i)-(a \cup b)$ so that $A \cap C(i)=\partial A \subseteq C(i)-(a \cup b)$ and $A$ is incompressible in $Q(i)-(a \cup b)$. Further suppose that the components of $\partial A$ are two sce which bound disjoint subdiscs $C^{\prime}$ and $C^{\prime \prime}$ of sphere $C(i)$ and annulus $A^{*}$ so that $C(i)=C^{\prime} \cup C^{\prime \prime} \cup A^{*}, A^{*} \cap C^{\prime}$ is one component of $\partial A$, and $A^{*} \cap C^{\prime \prime}$ is the other component of $\partial A$. $A \cup C^{\prime} \cup C^{\prime \prime}$ is a sphere which bounds subball $G(i) \subseteq Q(i)$ and $A \cup A^{*}$ bounds a "cube with hole" $W(i)=\overline{Q(i)-G(i)}$. Assume that each of the following is minimal: $\partial A \cap \sigma, \partial A \cap(\partial M(i) \cup \partial M(i+1)), D(i) \cap A, B(i) \cap A$, $F(i) \cap A, A \cap$ (boundaries of the 8 twisted rectangles of $F(i)$ and $B(i)$ ). Define collection $\Sigma=\{D(i) \cap A, B(i) \cap A, F(i) \cap A\}$.

The properties listed below follow in a straightforward manner.

(3.1) The components of each of $D(i) \cap A, B(i) \cap A, F(i) \cap A$ are sces which are noncontractible in $A$, and spanning arcs.

(3.2) Each component of $\partial A$ intersects $\sigma$ in exactly two points which lie in different segments: $\alpha, \beta, B(i) \cap M(i), B(i) \cap M(i+1)$.

(3.3) Each spanning arc of $\Sigma$ separates the surface $D(i), B(i)$ or $F(i)$ which contains it.

(3.4) There is at most one spanning arc of $\Sigma$ on $D(i)$, or on $F(i)$, and there are exactly two spanning arcs in $\Sigma$. (See (3.2).)

(3.5) Each spanning arc in $\Sigma$ meets $\overline{x y}$ in at most one point.

(3.6) $A$ can be deformed so that the sces of $\Sigma$ intersect each spanning arc of $\Sigma$ transversally in $A$, and in one point. Hence no surface $D(i), B(i), F(i)$ can have two scc of $\Sigma$, nor can it have both a spanning arc and a sce of $\Sigma$.

(3.7) There is at most one sec $\gamma$ in $\Sigma$ which must separate the surface $D(i), B(i)$, or $F(i)$ which contains it.

(3.7a) Simple closed curve $\zeta \subseteq F(i)$ which separates $F(i)$ is either a trefoil knot or bounds a disc in $F(i)$. If $\gamma \subseteq F(i), \gamma$ cannot be a trefoil knot, hence $\gamma$ would bound a subdisc of $F(i, 2)$ which contains $y$.

(3.7b) If $\gamma \subseteq D(i)$ then $\gamma$ would bound a subdisc of $D(i)$ which contains $x$.

(3.7c) The only scc $\zeta \subseteq B(i)$ which separates $B(i)$ is parallel in $B(i)$ to $\partial B(i)$ and meets $\overline{x y}$ in two points. But there is at most one 


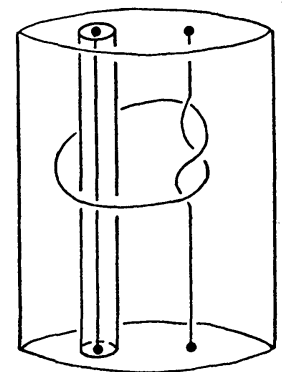

FIG. 9

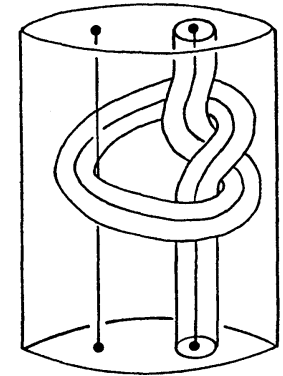

FIG. 10

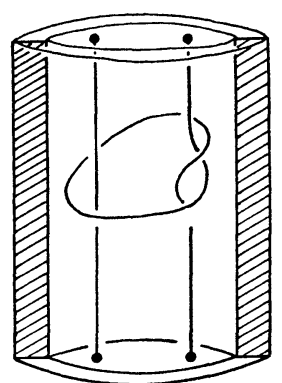

FIG. 11

spanning arc of $\Sigma$ in $D(i)((3.4),(3.5),(3.6))$. Hence $\gamma$ of $\Sigma$ cannot lie in $B(i)$.

From (3.0)-(3.7c) follows:

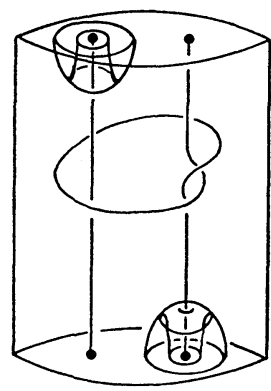

FIG. 12

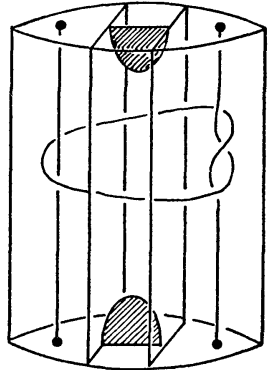

FIG. 13

Lemma 3.3. Under the hypotheses of (3.0), Figures 9, 10, 11, 12, 13 give the possible positions of $A$ in $Q(i)$ with the understanding that two additional variations for the position of $A$ which are similar to the two shown in Figure 12 are not illustrated in Figure 12.

Denote the two components of $D^{*} \cap \widetilde{Q}$ as subdiscs $D^{*}(a)$ and $D^{*}(b)$ where $a(i, 1) \cup a(i, 2) \subseteq D^{*}(a) \cap Q(i)=D^{*}(i, a)$ and $b(i, 1) \cup$ $b(i, 2) \subseteq D^{*}(b) \cap Q(i)=D^{*}(i, b), i=1,2,3$.

Lemma 3.4. Suppose annulus $A$ is as in (3.0) but with each instance of " $a \cup b$ " in (3.0) replaced by " $K$ ". Also let $a=a(i, 1)$ and let $a(i, 2)$ be parallel to $a(i, 1)$ in $D(i)$. Let $b=b(i, 1)$ and let $b(i, 2)$ be parallel to $b(i, 1)$ in $F(i)$. Then the possible positions for $A$ in $Q(i)-K$ are:

(1) as in Figure 9 with either one or both of $a(i, 1)$ and $a(i, 2)$ in $G(i)$;

(2) as in Figure 10 with either just one or both of $b(i, 1)$ and $b(i, 2)$ in $G(i)$; 
(3) as in Figure 11 with $K \subseteq G(i)$;

(4) as in Figure 13 with $K \cong G(i)$;

(5) eight possibilities similar to the two illustrated in Figure 12: four as in Figure 12 in which $A \cap D^{*}(a)=\varnothing=A \cap D^{*}(b)$ and four as in Figure 12 in which either $A \cap D^{*}(a) \neq \varnothing$ and $A \cap$ $D^{*}(b)=\varnothing$, or $A \cap D^{*}(a)=\varnothing$ and $A \cap D^{*}(b) \neq \varnothing$.

Proof. This follows from Lemma 3.3 and the observation that no component of $\partial A$ in $M(i)$ (or in $M(i+1)$ ) can intersect both $\partial D^{*}(a)$ and $\partial D^{*}(b)$. If this observation were not true such an intersection would give two spanning arcs of $A$, one in $D^{*}(a)$ which with $\alpha$ would bound a disc $D(i)$ in $Q(i)$, the other in $D^{*}(b)$ which with $\beta$ bounds a surface $F(i)$ in $Q(i)$. This would yield at one hand that $W(i)$ would be a "cube with unknotted hole" and at the other hand that $W(i)$ would be a "cube with knotted hole", a contradiction.

In preparation for Lemma 3.5 denote ball $\overline{S^{3}-\widetilde{Q}}$ by $V$. Note $C=\partial V$. Figure 14 illustrates $V$ and the four components of $V \cap K$. Singular disc $D^{*}$ intersects $V$ in three pieces: disc $D^{*}(1)$ bordered by segment $D^{*}(b) \cap M(1)$ and the single arc of $K$ cut by that segment, disc $D^{*}(3)$ bordered by segment $D^{*}(a) \cap M(4)$ and the single arc of $K$ cut by $D^{*}(a) \cap M(4)$, and disc $D^{*}(2)$ bordered by the segments $D^{*}(a) \cap M(1), D^{*}(b) \cap M(4)$, and the two so far unmentioned segments $K(1), K(2)$ of $K \cap V$. In the interior of $D^{*}(1)$ is segment $\overline{x(1) y(1)},\{x(1), y(1)\} \cong K$, of singularities of $D^{*}$ and the other segment $\overline{x(2) y(2)}$ of singularities of $D^{*}$ lies in the interior of $D^{*}(3)$. $D^{*}(2) \cap D^{*}(1)=\overline{x(1) y(1)}, D^{*}(2) \cap D^{*}(3)=\overline{x(2) y(2)}$.

Let disc $D \subseteq V$ be as illustrated in Figure 15. $D^{*}(2) \subseteq D$ and $\partial D$ consists of $K(1)$, segments $D^{*}(2) \cap(M(1) \cup M(4))$ and a simple arc

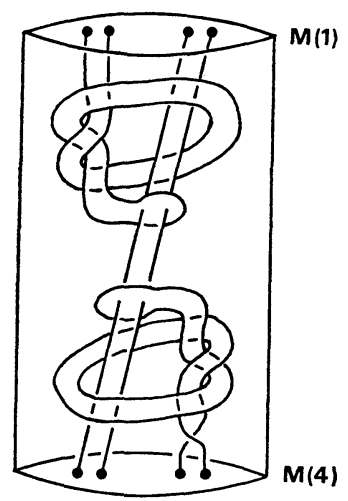

Fig. 14

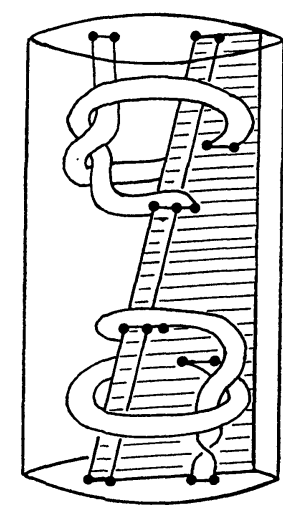

Fig. 15 
$\zeta$ which connects the ends of these two segments in $C$ and meets each of $\partial M(1)$ and $\partial M(4)$ once. $D$ meets $D^{*}(1)$ in two disjoint segments: $\overline{u(1) v(1)}$ in $D-\left(D^{*}(2) \cup \zeta\right)$, and $\overline{x(1) y(1) z(1)}$ where $Z(1) \epsilon$ $D^{*}(1) \cap\left(D-\left(D^{*}(2) \cup \zeta\right)\right)$. Analogous disjoint segments $\overline{u(2) v(2)}$, $\overline{x(2) y(2) z(2)}$ comprise $D \cap D^{*}(3)$.

Lemma 3.5. Suppose disc $E \subseteq V-K$ is such that $\partial E=E \cap$ $(C-K)$. Suppose that each of $D \cap E, D^{*}(1) \cap E, D^{*}(3) \cap E$ is minimal. As $E$ divides $V$ into subballs $Q^{\prime}, Q^{\prime \prime}$ such that $V=Q^{\prime} \cup Q^{\prime \prime}$, $Q^{\prime} \cap Q^{\prime \prime}=E$, then it must be that $D^{*} \cap V=D^{*}(1) \cup D^{*}(2) \cup D^{*}(3)$ is a subset of just one of the subballs $Q^{\prime}, Q^{\prime \prime}$.

Proof. Suppose not. Note that each arc: $\partial D^{*}(1), \partial D^{*}(3), K(1)$, $K(2)$ must lie entirely in $Q^{\prime}$ or in $Q^{\prime \prime}$.

(a) Suppose $\partial D^{*}(1) \subseteq Q^{\prime}$ and $K(1) \cup K(2) \cup \partial D^{*}(3) \subseteq Q^{\prime \prime}$. Then $D \cap E \neq \varnothing$. By the minimality of $D \cap E, \partial E \cap D=\varnothing$ so $D \cap E$ consists of disjoint sec $\gamma$ each of which bounds subdisc $D(\gamma)$ of $D$ which must contain at least one point of $\partial D^{*}(1) \cap D=\{u(1), v(1), z(1)\}$. Among such $\gamma$ there is a $D(\gamma)$ which contains no other sec of $D \cap E$. So $D(\gamma) \subseteq Q^{\prime}$. $\gamma$ also bounds subdisc $E(\gamma)$ of $E$ where $E(\gamma) \cap K=\varnothing$ and $E(\gamma) \cap D(\gamma)=\gamma$. So sphere $E(\gamma) \cup D(\gamma)$ bounds subball $V^{*}$ in the interior of $V$. Of the three points $u(1), v(1), z(1)$, just one cannot lie in $D(\gamma)$, yet if two are in $D(\gamma)$, the third is isolated for another instance of just one point of $\partial D^{*}(1)$ in some other "innermost" $D(\gamma)$. Yet all there of $u(1), v(1), z(1)$ cannot lie in $D(\gamma)$. Hence case (a) cannot occur. Similarly

(b) $\partial D^{*}(3) \cong Q^{\prime}$ and $K_{1} \cup K_{2} \cup \partial D^{*}(1) \subseteq Q^{\prime \prime}$ cannot occur.

(c) Suppose $K(1) \subseteq Q^{\prime}, K(2) \subseteq Q^{\prime \prime}$. By the minimality of $D \cap E$ there is one spanning arc of $D \cap E=D^{*}(2) \cap E$, with ends in the two components of $D^{*}(2) \cap C$.

(i ) $\partial D^{*}(1) \cup \partial D^{*}(3) \cong Q^{\prime}$. To separate $K(2)$ from the six points $u(1), u(2), v(1), v(2), z(1), z(2)$, each point must lie in subdisc $D(\gamma)$ of $D$ cut by scc $\gamma \subseteq E \cap D$ which contains no other sec of $E \cap D$. There could be one, two, three-up to six such subdiscs $D(\gamma)$, but each combination leaves a situation similar to case (a): an odd number of the six points in the boundary of a ball in the interior of $V$, a contradiction.

(ii) $\partial D^{*}(1) \subseteq Q^{\prime}, \partial D^{*}(3) \subseteq Q^{\prime \prime}$, or $\partial D^{*}(3) \subseteq Q^{\prime \prime}, \partial D^{*}(1) \subseteq Q^{\prime}$ each gives a case similar to (i) above.

(iii) $\partial D^{*}(1) \cup \partial D^{*}(3) \subseteq Q^{\prime \prime}$. Redefine disc $D$ so that $K(2) \subseteq \partial D$ instead of $K(1)$, then apply (i) and (ii) above.

(d) Suppose $K(1) \cup K(2) \subseteq Q^{\prime}$ and $\partial D^{*}(1) \cup \partial D^{*}(2) \subseteq Q^{\prime \prime}$. Again, there is just one spanning arc of $E \cap D$ which separates $K(1) \cup K(2)$ from $u(1), u(2), v(1), v(2), z(1), z(2)$. It can be assumed that the span- 
ning arc meets each of $\overline{x(1) y(1)}$ and $\overline{x(2) y(2)}$ in a single point. So $E \cap D^{*}(1) \neq \varnothing \neq E \cap D^{*}(3)$. Each of these two intersections consists of sec $\gamma$. Among such $\gamma$ on $D^{*}(1)$ is an "innermost" $\gamma$ which bounds subdisc $D(\gamma)$ of $D^{*}(1)$ which contains no other sce of $E \cap D^{*}(1)$ yet which contains a point of $\{x(1), y(1)\} . \quad \gamma$ bounds subdisc $E(\gamma) \subseteq E$. If $E(\gamma) \cap D^{*}(3)=\varnothing$ then sphere $D(\gamma) \cup E(\gamma)$ bounds a ball $V^{*}$ in the interior of $V$ and $\partial V^{*}$ contains just one point of $K(1)$ or $K(2)$, which is impossible. So $E(\gamma) \cap D^{*}(3) \neq \varnothing$. There exists scc $\gamma(1)$ in $E(\gamma) \cap D^{*}(3)$ which bounds "innermost" subdisc $D(\gamma(1))$ of $D^{*}(3)$ which contains a point of $\{x(2), y(2)\} . \quad \gamma(1)$ bounds a subdisc $E(\gamma(1)) \subseteq E(\gamma)$ for which, for the same reason two sentences above, $E(\gamma(1)) \cap$ $D^{*}(1) \neq \varnothing$. Thus there is an infinite sequence of disjoint sec $\gamma, \gamma(1)$, $\gamma(2), \cdots$ of $E \cap\left(D^{*}(1) \cup D^{*}(3)\right)$ bounding properly nested subdiscs $E \supset E(\gamma) \supset E(\gamma(1)) \supset E(\gamma(2)), \ldots$ which is impossible. Cases (a), (b), (c), (d) cover all possible negations of the conclusion of Lemma 3.5 and these cases cannot occur. So Lemma 3.5 is proved.

The discussion continues in the context of Lemma 3.5. Three more discs $M(5), M(6), M(7)$ are determined in $V$ as in Figure 16. Note their properties: $D^{*}(2) \cap M(7)=\overline{x(1) y) 1)}, D^{*}(2) \cap M(5)=\overline{x(2) y(2)}$, $D^{*}(1) \cap M(7)$ is a subdisc of $D^{*}(1)$ which contains $\overline{x(1) y(1) z(1)}, D^{*}(3) \cap$ $M(5)$ is a subdisc of $D^{*}(3)$ which contains $\overline{x(2) y(2) z(2)}, D^{*}(2) \cap M(6)=$ $D^{*} \cap M(6)$ is a spanning arc of $D^{*}(2)$ which separates $D^{*}(2)$ into two subdiscs, one containing $\overline{x(1) y(1)}$, the other containing $\overline{x(2) y(2)} . Q(4)$ denotes the subball cut from $V$ by $M(4)$ and $M(5)$, ball $Q(5)$ is cut from $V$ by $M(5)$ and $M(6), Q(6)$ is cut by $M(6)$ and $M(7), Q(7)$ is cut by $M(7)$ and $M(1)$. In $Q(4)$ determine Möbius band $B(4)$ and in $Q(7)$ determine Möbius band $B(7)$ as illustrated in Figure 17. $\partial B(4)$ consists of a center line in $D^{*}(3)$ from $D^{*}(3) \cap M(4)$ to $x(2)$, the segment $K(1) \cap Q(4)$ to $M(4)$, and segment $B(3) \cap M(4)$. $\partial B(7)$ similarly consists of a center line in $D^{*}(1)$ from $D^{*}(1) \cap M(1)$ to $x(1)$, the seg-

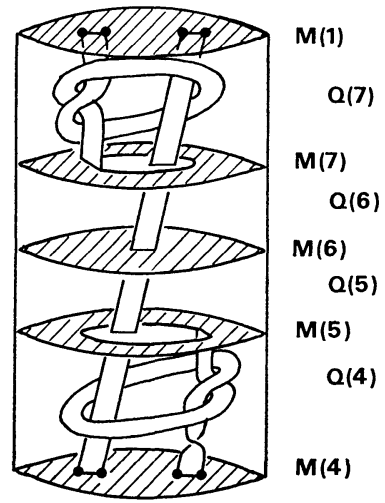

FIG. 16

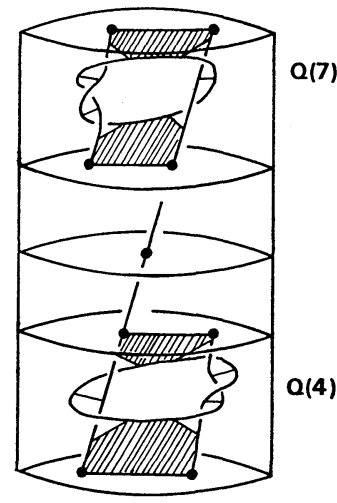

FIG. 17 
ment $K(1) \cap Q(7)$, and segment $B(1) \cap M(1)$. Each of $B(4)$ and $B(7)$ consists of two discs connected by the five twisted rectangles as shown.

4. $K$ is simple. Let $T$ be a torus which is incompressible in $S^{3}-K$. Denote the component of $S^{3}-T$ which contains $K$ by $G$. $T \cap C$ consists of a collection of disjoint simple closed curves. Suppose $T \cap(M(2) \cup M(3))$ is minimal, and $T \cap C$ is minimal. Then, by Lemma 3.2 and Lemma 3.5, no sce of either $T \cap C$ or $T \cap(M(2) \cup$ $M(3)$ ) can bound a disc in $\widetilde{Q}$ or in $V$. So the closures of components of $T-(C(1) \cup C(2) \cup C(3))$ are incompressible annuli in $Q(1)-K$, $Q(2)-K, Q(3)-K, V-K$. Denote this collection of annuli by $\Gamma$. Suppose $\Gamma \neq \varnothing$. The annuli of $\Gamma$ can be positioned so as to satisfy the hypotheses of Lemmas 3.3 and 3.4. Then, from the minimality conditions three sentences above and from the incompressibility of $T$ in $S^{3}-K$, none of the Lemma 3.4 cases (3), (4), (5) can occur in $Q(i), i=1,2,3$. So if $\Gamma \neq \varnothing$ it can be assumed that $T \cap(C-(M(1) \cup$ $M(4)))=\varnothing . \quad T$ can be deformed so that $T \cap D^{*}$ is minimal. Suppose annulus $A \in \Gamma, A=A(i) \subseteq T \cap Q(i), i \in\{1,2,3\}$, is an instance of (1) of Lemma 3.4 for which $D^{*}(i, a) \cong G(i)$. $A$ gives rise to two more annuli of $\Gamma$ of the same type of case (1) of Lemma 3.4 in the other two balls of $\widetilde{Q}$. The three annuli join together in $T$ to give an incompressible annulus $A^{*}$ of $T \cap \widetilde{Q}$ which forms part of the boundary of ball $G(1) \cup G(2) \cup G(3)=G^{*}$ where $D^{*}(a) \subseteq G^{*}$ and $D^{*}(b) \subseteq \widetilde{Q}-G^{*}$. Thus $\partial D^{*}(2) \cap M(1) \subseteq \partial G^{*}$ and $\partial D^{*}(3) \cap M(4) \subseteq \partial G^{*}$ but segments $D^{*}(b) \cap(M(1) \cup M(4))$ are not in $\partial G^{*}$. In $Q(4)$ annulus $\widetilde{A}$ of $T \cap Q(4)$ which shares the component $\partial A \cap M(4)$ with $A^{*}$ must be part of a ball $\widetilde{G}$ in $Q(4)$ which contains $\partial D^{*}(3)$ but does not contain $(K(1) \cup K(2)) \cap Q(4)$. Then $\widetilde{G} \cap M(5)$ is a disc, bounded by a component of $\partial \widetilde{A}$, which contains no point of $K(1) \cup K(2)$. As $T-$ $(C-(M(1) \cup M(4)))=\varnothing$, the component $\widetilde{A}^{\prime}$ of $T \cap Q(5)$ continuing from $\widetilde{A}$ must have a boundary component in $Q(6)$. The ball $\widetilde{G}^{\prime}$ cut by $\widetilde{A}^{\prime}$ from $Q(5)$ which shares disc $\widetilde{G} \cap M(5)$ with $\widetilde{G}$ can contain no points of $K(1) \cup K(2)$. $\quad \widetilde{G}^{\prime} \cap M(6)$ is a subdisc of $M(6)$ which has no points of $K$, hence $T$ would be compressible in $S^{3}-K$, a contradiction. So there can be no such $A$ above and the only instances of (1) from Lemma 3.4 which arise are those for which just one of $a(i, 1), a(i, 2)$ is contained in $G(i)$. Similarly the only instance of (2) from Lemma 3.4 which can occur are those for which just one of $b(i, 1), b(i, 2)$ is contained in $G(i)$. From this easily follows:

LEMMA 4.1. If $\Gamma \neq \varnothing, T$ bounds a regular neighborhood of $K$.

Next, suppose that $\Gamma=\varnothing$. There is no loss in generality to 
assume that $T \cap C=\varnothing$ and $T \cap\left(\bigcup_{k=1}^{7} M(k)\right)=\varnothing$. Then $T \subseteq Q(i)-K$ for some $i \in\{1,2,3,4,5,6,7\}$. As $T$ is incompressible in $S^{3}-K$ it may also be assumed that $T \cap\left(D^{*} \cap Q(i)\right)=\varnothing$. If $W$ is the component of $S^{3}-T$ other than $G$, then $\bar{W} \leqq Q(i)-\left(\partial Q(i) \cup D^{*}\right)$.

Suppose $i \in\{1,2,3,4,7\}$. Let $P$ be a disc properly embedded in $Q(i)$ with $\partial P=\sigma\left(\right.$ cf (3.2)) so that $P$ contains as subdiscs $D(i), D^{*}(i, a)$, $F(i, 1), B(i, 1), B(i, 2)$, and two pieces of $D^{*}(i, b) . \quad P$ intersects the rest of $D^{*}(i, b)$ in three segments, oue in $D(i)$, two in $F(i, 1)$. It can be assumed that $T \cap P$ is minimal. As $T$ is in the interior of $Q(i)$ and separates $Q(i), T \cap P$ must have as components at least 4 of the 5 scc illustrated by dashed lines in Figure 18. Select the two subdiscs $P(1), P(2)$ of $P$ as in Figure $19, P(1) \subset P(2) \subset P$. As $P(1)$ is pivoted in $Q(i)$ on its edge in $D^{*}(i, a)$ through angles $\theta$ : $|\theta| \leqq 5 \pi / 6$, the components of $D(i, \theta) \cap T$ sweep out two annuli in $T$ and part of the boundary of a regular neighborhood of $D^{*}(i, a)$ in $Q(i)$, under the assumption that $T \cap P(1, \theta)$ is minimal, for each $\theta$. See Figure 20. Similarly as $P(2)$ is pivoted in $Q(i)$ on its edge in $D^{*}(i, b)$, as in Figure 21, through $\theta:|\theta| \leqq \pi / 2$, the sweep continues to complete the tube around $D^{*}(i, a)$, again, under the assumption that $T \cap P(2, \theta)$ is minimal, each $\theta$. At $|\theta|=\pi / 2$, the $P(2, \theta)$ 's have

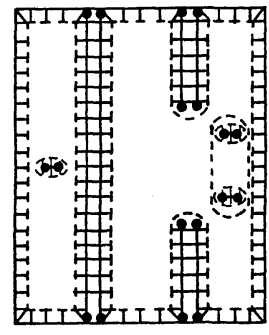

FIG. 18

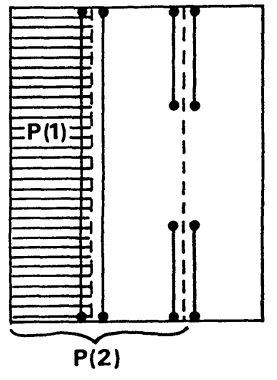

FIG. 19

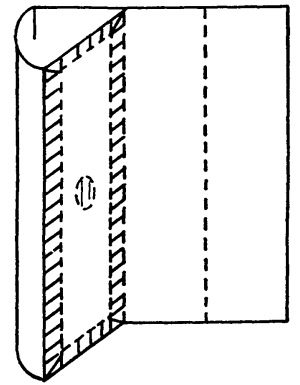

Fig. 20

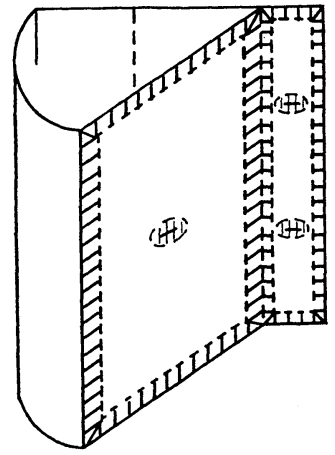

FIG. 21

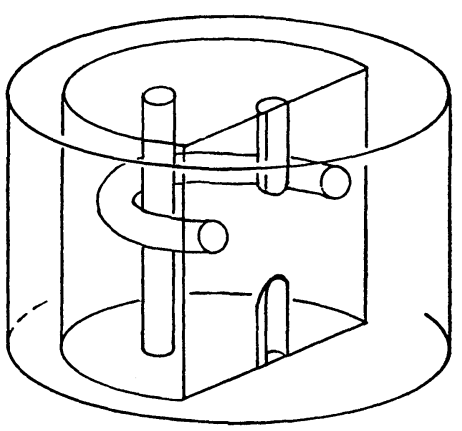

FIG. 22 
swept the part of $T$ in Figure 22. For $\pi / 2 \leqq|\theta| \leqq \pi$ the family $\{P(2, \theta)\}$ complete $T$ to yield a surface of genus two as the only candidate for $T$, a contradiction.

For $i \in\{5,6\}$ and $T \cap\left(D^{*} \cap Q(i)\right)=\varnothing, T \leqq Q(i)$ is impossible as there are no incompressible surfaces in $Q(i)-D^{*}, i=5,6$.

Hence one can conclude:

LEMMA 4.2. $\quad \Gamma \neq \varnothing$.

Lemma 4.1 and Lemma 4.2 then give

THEOREM $43 . K$ is simple.

5. $K$ is of genus one. Suppose $i \in\{1,2,3,4,7\}$ and recall Möbius band $B(i)$ in $Q(i)$ as presented earlier. Suppose disc $E \subseteq Q(i)$ is such that $\partial E=\partial B(i)$. Consider disc $P$ of Lemma 4.2 for which Figure 23 features $D(i), a \subseteq P$, and $b \cap P$ consisting of two segments and three points, one of which is $b \cap D(i)=\{x\}$. Again let $\{P(2, \theta)\}$ be the family of dises of which $P(2, \theta)$ is obtained by pivoting $P(2)=P(2,0)$ on its edge which contains the two arcs of $b$ through angle $\theta,|\theta| \leqq \pi$. (See Figure 23.) Assume $E \cap P(2, \theta)$ is minimal, for each $\theta$. For $0<|\theta|<5 \pi / 6$ each point $x(\theta)$ must connect by an arc of $E \cap P(2, \theta)$ to a point on the pivot edge of $P(2)$. Yet at $\theta=0, X(0)$ in $D(i)$ connects by an arc of $E \cap D(i)$ to a point of $a$. This is impossible. Consequently, one has:
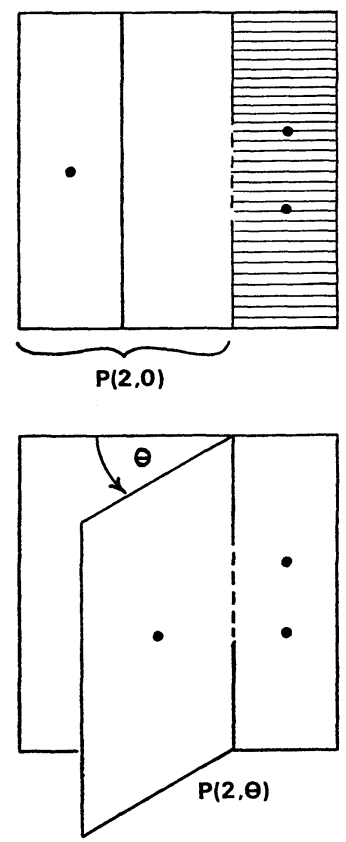

FIG. 23 
Lemma 5.1. For $i \in\{1,2,3,4,7\}, \partial B(i)$ cannot bound a disc in $Q(i)$.

REMARK 5.1. For $i \in\{1,2,3\}$, if disc $E \subseteq Q(i)$ is such that $E \cap C(i)=\operatorname{arc} \alpha^{\prime}, \partial E=a \cup \alpha^{\prime}, \alpha^{\prime} \cap b=\varnothing$ and if $E \cap b$ is minimal, then $(E-\partial E) \cap(b-\partial b) \neq \varnothing$.

REMARK 5.2. For $i \in\{1,2,3\}$, if arc $\beta^{\prime} \subseteq C(i)$ is such that $\beta^{\prime} \cap b=\partial \beta^{\prime}=\partial b$, then $\beta^{\prime} \cup b$ cannot bound a disc in $Q(i)$.

REduction Remark 1. Suppose $E$ is a compressing disc for (possibly bordered) surface $F$ (i.e., $E \cap F=\partial E$ and $\partial E$ does not bound a disc on $F$ ). Suppose $s$ and $t$ are two points of $\partial E$ and simple arc $\delta \subseteq E$ is such that $\partial \delta=\{s, t\}$ and $\delta$ cuts $E$ into two subdiscs, $E^{\prime}, E^{\prime \prime}$. Suppose simple arc $\eta \subseteq F$ is such that $\partial \eta=\{s, t\}$ and there exists disc $E^{*}$ for which $\partial E^{*}=\delta \cup \eta, E^{*} \cap E=\delta, E^{*} \cap F=\eta$, then one of $\operatorname{discs} E^{\prime} \cup E^{*}$ or $E^{\prime \prime} \cup E^{*}$ is a compressing disc for $F$.

REDUCTION REMARK 2. If $E$ is a compressing disc for surface $F$, sec $\gamma \subseteq E-\partial E$ bounds disc $E(\gamma) \subset E, \gamma$ bounds disc $E^{*}$ such that $E^{*} \cap(E-E(\gamma))=\varnothing, E^{*} \cap F=\varnothing$, then $(E-E(\gamma)) \cup E^{*}$ is a compressing disc for $F$.

Theorem 5.1. $S(-1)$ is incompressible. $K$ is of genus one.

Proof. Suppose $E$ is a compressing disc for $S(-1)$. Assume for each $i \in\{1,2,3,4,5,6\}$ that $E \cap C(i)$ is minimal not only via deformations but also with respect to the operations of the two reduction remarks. Also assume that $\partial E \cap\left(\bigcup_{k=1}^{7} M(k)\right)$ is minimal.

If $\partial E \cap D^{*} \neq \varnothing$ then $E \cap C \neq \varnothing$ and for $i \in\{1,2,3\}, E \cap C(i) \neq \varnothing$. Because of Lemma 3.2 the components of $E \cap C(i)$ are subdises $E^{\prime}$ of $E, E^{\prime} \cap C(i)=\partial E^{\prime}$, where $\partial E^{\prime}$ is composed of simple arcs in $C(i)$, with ends in some of the four segments of $\left(D^{*}(i, a) \cup D^{*}(i, b)\right) \cap$ $(M(i) \cup M(i+1))$, and of center lines of $D^{*}(i, a)$ or $D^{*}(i, b)$. Just three possibilities need be considered.

(a) $\partial E^{\prime}=a \cup \alpha^{\prime}$ where $a$ is a center line of $D^{*}(i, a)$ and $\alpha^{\prime} \subseteq$ $C(i)$. By Remark 5.1 this case cannot occur.

(b) $\partial E^{\prime}=b \cup \beta^{\prime}$ where $b$ is a center line of $D^{*}(i, b)$ and $\beta^{\prime} \subseteq C(i)$. By Remark 5.2 this cannot occur.

(c) $\partial E^{\prime} \cap D^{*}(i, a)=a$, a centerline of $D^{*}(i, a) ; \partial E^{\prime} \cap D^{*}(i, b)=$ $b, a$ centerline of $D^{*}(i, b)$; there exists arc $\alpha^{\prime}$ in $M(i+1)$ from $\partial a$ to $\partial b$; there exists arc $\beta^{\prime}$ in $M(i)$ from $\partial a$ to $\partial b$, so $E^{\prime} \cap C(i)=\alpha^{\prime} \cup \beta^{\prime}$; $\partial E^{\prime}=a \cup \alpha^{\prime} \cup b \cup \beta^{\prime}$. But then $\partial E^{\prime}$ bounds a Möbius band similar to $B(i)$ in $Q(i)$. This is contrary to Lemma 5.1 . 
As none of (a), (b), (c), can occur, $\partial E \cap D^{*}=\varnothing$. Thus $\partial E \subseteq H$. It can be assumed that $\partial E=H \cap M(6)$ which bounds subdisc $M^{*}$ of $M(6)$ and $M^{*} \cap D^{*}(2)$ is a segment. There is no loss of generality to assume that $E \cap M^{*}=\partial E$ and that $E \cup M^{*}$ is a sphere in ball $Q(5) \cup Q(6)$. But $E \cup M^{*}$ contains just one point of $K(1)$, a contradiction. So, there can be no compressing disc for $S(-1)$ and $S(-1)$ is incompressible in $S^{3}-K . K$ is of genus at least one, and $S(-1)$ then guarantees that $K$ is of genus one.

6. More spanning surfaces for $K$. Recall that annulus $H$ bounded a regular neighborhood of $D^{*}(2) \cap(Q(5) \cup Q(6))$. Figure 24 shows $H$ continued through $Q(4), Q(7)$ and $\widetilde{Q}$ to yield $\widetilde{H}$, part of the boundary of a regular neighborhood of disk $D^{* *}=D^{*}(2) \cup D^{*}(a) \cup$ $D^{*}(b)$. Cap annulus $\tilde{H}$ by the disc in $M(1)$ cut by $\partial \widetilde{H}$, which contains $D^{*}(b) \cap M(1)$, and by the disc in $M(4)$ cut by $\partial \widetilde{H}$ which contains $D^{*}(a) \cap M(4)$. The result is a sphere $Z$ which bounds 3-cell $U$, as in Figure 24.

For $i \in\{1,2,3\}$ define $C^{*}(i)=\overline{C(i)-U, Q^{*}}(i)=\overline{Q(i)-U \text {, }}$ so that

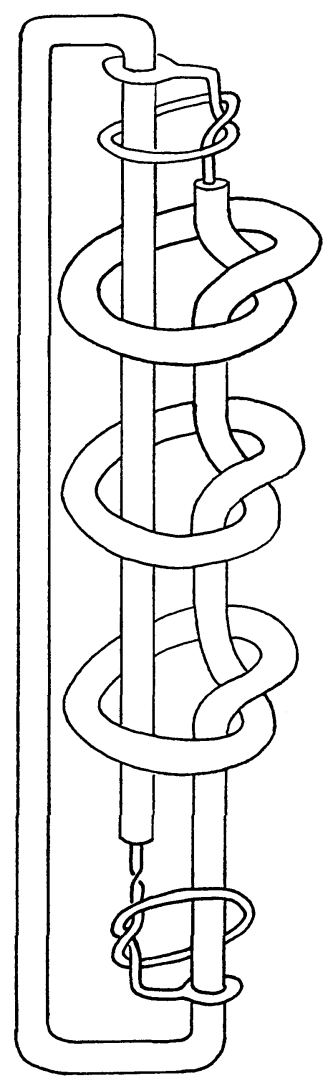

FIG. 24 
$C^{*}(i)$ is a sphere with four holes and $Q^{*}(i)$ is a "cube with two holes".

Next, restrict $i \in\{2,3\}$. Consider small product neighborhood $C(i) \times I$ of $C(i)$ in $Q(i)$, where $I=[0,1]$. Let $n \in\{1,2,3, \cdots\}$ and define $C(2, j)=C(2) \times\{j / n\}$ for $j \in\{0,1,2,3, \cdots, n\}, C(3, k)=C(3) \times$ $\{(k-1) /(n+1)\}, k \in\{1,2, \cdots, n+1\}$. Note that $C(2)=C(2,0)$, that there are $n$ additional copies of $C(2)$ in the interior of $Q(2)$, each copy parallel to $C(2)$ in $Q(2)$. Also, $C(3,1)=C(3) \times\{0\}=C(3)$, but $C(2,1)=C(2) \times\{1 / n\} \neq C(2)$. Note also that the elements of $\{C(1)$, $C(2, j), C(3, k) ; j=1,2, \cdots, n, k=1,2, \cdots, n+1\}$ are disjoint.

Disc $D^{* *}$ is cut by $C^{*}=C(1) \cup \bigcup_{j=1}^{n} C(2, j) \cup \bigcup_{k=1}^{n+1} C(3, k)$ in $8 n+8$ disjoint spanning segments which cut $4 n+4$ properly nested sub$\operatorname{discs} D^{* *}(1) \supset D^{* *}(2) \supset \cdots \supset D^{* *}(4 n+4)$, where $D^{* *}(1)=D^{* *}$. Note also that $C^{*} \cap U$ is a collection of $8 n+8$ disjoint dises $L(t), t=$ $1,2, \cdots, 8 n+8$, parallel in $U$ and which slice $\tilde{H}$ into $8 n+7$ annuli $H(k), k \in\{1,2, \cdots, 8 n+7\}$. For $\iota \in\{1,2, \cdots, 4 n+4\}$ first note that $\partial D^{* *}(\ell) \subseteq L(\ell) \cup L(8 n+8-\ell+1)$. Secondly, let $U(\ell)$ be the component of $U$ cut by the dises $L(\ell), L(8 n+8-\ell+1)$ with these discs attached. So $D^{* *}(\ell) \subseteq U(\ell)$. Define $\widetilde{H}(\zeta) \subseteq \widetilde{H}$ such that $\partial U(\ell)=L(\ell) \cup \widetilde{H}(\ell) \cup L(8 n+8-\ell+1)$. In $U-D^{* *}$ consider product neighborhood $\widetilde{H} \times I$ of $\widetilde{H}$. Then for $\iota \in\{1,2, \cdots, 4 n+4\}$ define $H^{\prime}(\ell)=\tilde{H}(\ell) \times\{(4 n+4-\ell) /(4 n+4)\}$. Next attach the annuli cut from each of discs $L(\ell)$ and $L(8 n+8-\ell+1)$ by $\partial H^{\prime}(\ell)$, to $H^{\prime}(\ell)$ to form annulus $H^{*}(\ell)$. $H^{*}(\ell)$ is merely $H^{\prime}(\ell)$ which is "flared" so that its boundary lies in $\tilde{H}$. The "flare" disappears at $H^{*}(4 n+4)=\widetilde{H}(4 n+4) \times\{0\} \leqq \widetilde{H} . H^{*}(4 n+4)$ lies in $V$ and $H=$ $H^{*}(4 n+4) \cap(Q(5) \cup Q(6))$. Define $C^{*}(2, j)=\overline{C(2, j)-U}, j=1,2, \cdots$, $n, C^{*}(3, k)=\overline{C(3, k)-U, k}=1,2, \cdots, n+1$. Let $F^{*}(n)=C^{*}(1) \cup$ $\bigcup_{j=1}^{n} C^{*}(2, j) \cup \bigcup_{k=1}^{n+1} C^{*}(3, k) \cup \bigcup_{c=1}^{4 n+4} H^{*}(\ell)$.

Each of the four boundary components of each of $C^{*}(1), C^{*}(2, j)$, $C^{*}(3, k), j=1,2, \cdots, n, k=1,2, \cdots, n+1$, is a boundary component of just one $H^{*}(\ell)$ and so $F^{*}(n)$ is a single closed surface. Figure 25 schematically illustrates the connection scheme for $F^{*}(n)$. The scheme is similar to the one in [2] but the surface $F^{*}(n)$ here is actually connected in a different manner. The surfaces $F^{*}(n)$ are adapted from one of the algorithms in [1]. Euler characteristic:

$$
\begin{aligned}
\chi\left(F^{*}(n)\right) & =\sum_{j=1}^{n} \chi\left(C^{*}(2, j)\right)+\sum_{k=1}^{n+1} \chi\left(C^{*}(3, k)\right)+\chi\left(C^{*}(1)\right) \\
& =n(-2)+(n+1)(-2)+(-2)=-4(n+1) .
\end{aligned}
$$

Hence $F^{*}(n)$ has genus $2 n+3$. If $n=0$ were allowed, then $F^{*}(0)=$ $C^{*}(1) \cup C^{*}(3) \cup \bigcup_{c=1}^{4} H^{*}(\ell)$ is again a surface of the same type as $F^{*}(n), n=1,2, \cdots$, and has genus 3 . So, in general, $n \in\{0,1,2, \cdots\}$ 


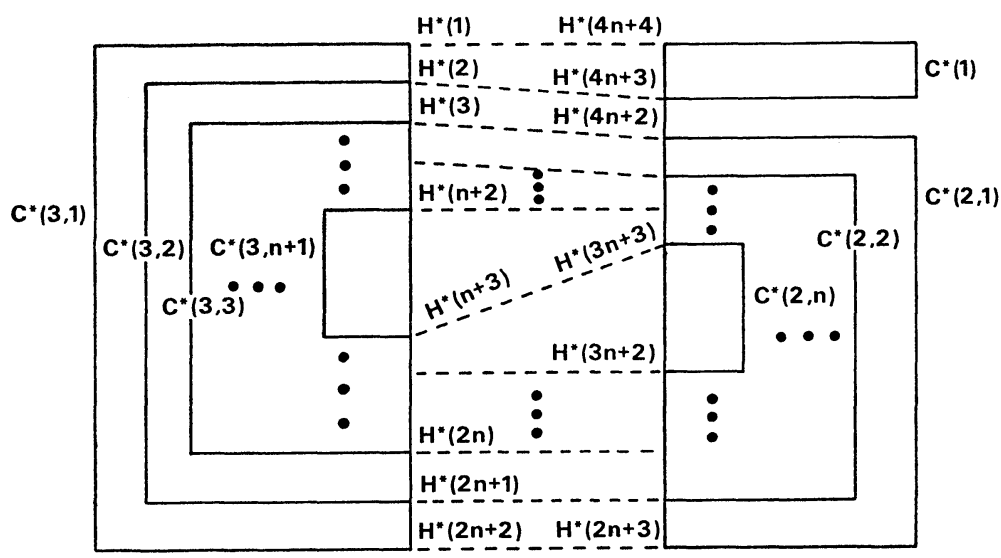

FIG. 25

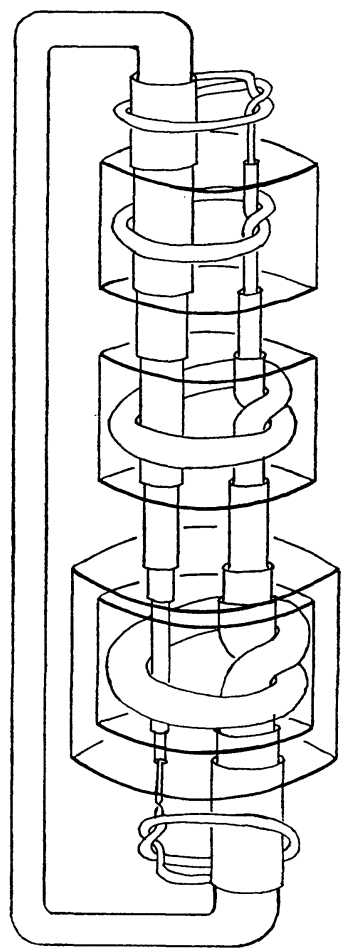

FIG. 26

is permitted. Each $F^{*}(n)$ is a closed surface in $S^{3}$, hence $F^{*}(n)$ is orientable.

Remove $H$ from $F^{*}(n)$ to obtain orientable spanning surface $\left.S(n)=\overline{\left(F^{*}(n)-H\right)} \cup \overline{\left(D^{*}-\left(D^{\prime} \cup D^{\prime \prime}\right)\right.}\right)$ for $K$, of genus $2 n+3, n=$ $0,1,2, \cdots$ Figure 26 illustrates $S(1)$.

Lemma 6.1. For each $\ell=1,2, \cdots, 4 n+4, H^{*}(\iota)$ is incompres- 
sible in $S^{3}-K$.

Proof. Each noncontractible sec $\gamma$ in $H^{*}(\ell)$ is parallel in $H^{*}(\ell)$ to either component of $\partial H^{*}(\ell)$, hence $\gamma$ bounds a disc in $U$ which intersects each of the two arcs of $K \cap U$ in one point. If such a sec $\gamma$ were to bound a disc $E$ in $S^{3}-K, E \cap H^{*}(\ell)=\gamma$, a contradiction similar to that in the proof of Lemma 3.1 would arise.

Lemma 6.2. For each $i=1,2,3, C^{*}(i)$ is incompressible in $S^{3}-K$.

Proof. Suppose not. Suppose $E$ is a compressing disc for $C^{*}(i)$, some $i \in\{1,2,3\}$. Then $E \cap C^{*}(i)=\partial E$ does not bound a disc on $C^{*}(i)$. Assume that $E \cap\left(\bigcup_{j \neq i}\left(C^{*}(j)-C^{*}(i)\right)\right)$ is minimal not only with respect to deformations but also with respect to the two reduction remarks. Then, by Lemmas 3.2 and 3.5, $E \subseteq Q_{*}(i)$. But this contradicts Lemma 3.2.

TheOREM 6.1. For $n \in\{0,1,2, \cdots\}, S(n)$ is incompressible in $S^{3}-K$.

Proof. Suppose not. Suppose $E$ is a compressing disc for $S(n)$, some $n \in\{0,1,2, \cdots\}$. Then $E \cap S(n)=\partial E$ does not bound a disc on $S(n)$. Assume for each $i=1,2,3,4,5,6,7$ that $E \cap C(i)$ is minimal, and that $E \cap \tilde{H}$ is minimal.

If $E \cap \widetilde{H}=\varnothing$, then just one of the following can hold:

(a) there is some $\ell \in\{1,2, \cdots, 4 n+4\}$ such that $\partial E \subseteq H^{*}(\ell)$,

(b) either $\partial E \subseteq C^{*}(1)$ or there is some $i \in\{2,3\}$, some $j \in$ $\{1,2, \cdots, n+1\}$ such that $\partial E \subseteq C^{*}(i, j)$,

(c) $\partial E \cong D^{*}-\left(D^{\prime} \cup D^{\prime \prime}\right)$.

(a) and (b) cannot occur because of Lemma 6.1 and Lemma 6.2. In the proof of Theorem 5.1 it was shown that (c) cannot hold. So $E \cap \widetilde{H} \neq \varnothing$. Note that $\widetilde{H} \cap(Q(4) \cup Q(7))=\overline{H^{\prime}(4 n+4)-H} \subseteq S(n)$ so if $E \cap\left(\overline{H^{\prime}(4 n+4)-H}\right) \neq \varnothing, E \cap\left(\overline{H^{\prime}(4 n+4)-H}\right) \subseteq \partial E$. Let collection $O^{*}$ consist of annuli $H(k), k=1,2,3, \cdots, 8 n+7$ with the one identified with $H^{\prime}(4 n+4)$ removed and replaced by $H$ (so that there still are $8 n+7$ of them) and two discs $L^{*}(1), L^{*}(8 n+8)$, where $L^{*}(1)$ is cut from $L(1)$ by $\partial H^{\prime}(1)$ and $L^{*}(8 n+8)$ is cut from $L(8 n+8)$ by $\partial H^{\prime}(1)$. Denote segment $c=D^{* *} \cap L^{*}(1)$, segment $d=$ $D^{* *} \cap L^{*}(8 n+8)$.

From minimality conditions above, for each $H(k) \in O^{*}, H(k) \cap E$ consists of at most disjoint spanning ares in $H(k)$ and $E$ with boundary in both components of $\partial H(k)$. Each of $E \cap L^{*}(1)$ and $E \cap$ 


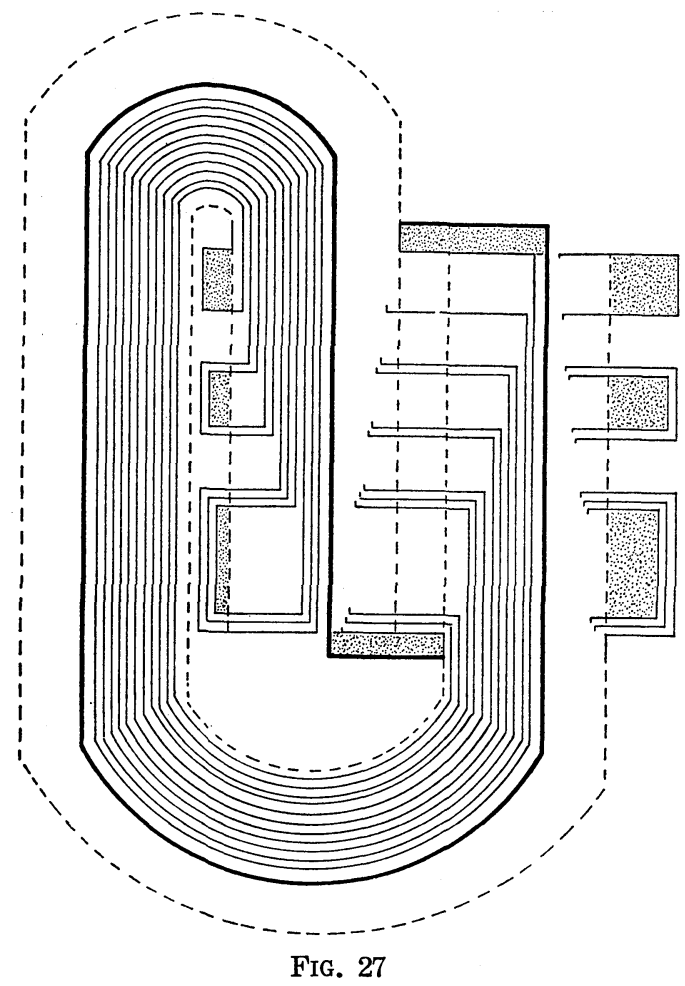

$L^{*}(8 n+8)$ consists of at most spanning arcs in $E$ with boundary in $\partial L^{*}(1)$ and $c$, or in $\partial L^{*}(8 n+8)$ and $d$. Call the class of all such $\operatorname{arcs} \widetilde{O}$ and suppose $\widetilde{O} \neq \varnothing$. Then each arc in $\widetilde{O}$ separates $E$ into two disjoint subdises and among these subdises are "end-dises" which contain no other subdisc. If $\hat{O} \neq \varnothing, E$ must have at least two "end-discs". If $E^{*}$ is an "end-disc", then $\partial E^{*}=\delta \cup \eta$ where $\delta$ is a spanning arc in $\widetilde{O}$ and $\eta \cong \partial E$. There are just two possibilities for $\delta$ :

(a) $\delta \subseteq H(k)$, and $\eta$ is a subset of one of $C^{*}(1), C^{*}(2, n)$, $C^{*}(3, n+1)$, and $E^{*} \subseteq Q^{*}(1)$, or $E^{*} \subseteq Q^{*}(2, n)$, or $E^{*} \subseteq Q^{*}(3, n+1)$;

(b) $\delta \leqq L^{*}(1)$ and $\eta \cong C^{*}(1) \cup D^{*}(1) \cup(\widetilde{H} \cap Q(7))$ with one arc in each of the named pieces, and $E^{*} \subseteq Q(7)$ or $\delta \subseteq L^{*}(8 n+8)$ and $\eta \subseteq C^{*}(3) \cup D^{*}(3) \cup(\tilde{H} \cap Q(4))$ and $E^{*} \subseteq Q(4)$.

Figure 27 gives a schematic representation of $S(2)$ with possibilities (a) and (b) denoted by "speckled" discs. Case (a) contradicts Remark 5.1. or Remark 5.2 and case (b) contradicts Lemma 5.1. So $\widetilde{O}=\varnothing$, which contradicts $E \cap \widetilde{H} \neq \varnothing$. Hence $S(n)$ is incompressible in $S^{3}-K$.

7. A conjecture. Theorems 4.2. 5.1, 6.7 establish the existence of a knot as in the title of this paper. If one were to replace arc 


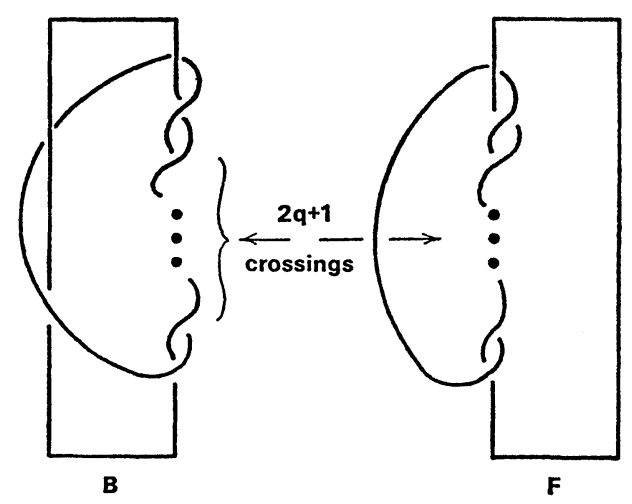

FIG. 28

$b$ in $Q(2)$, which has 3 self-crossings which provide $F(2)$ and $B(2)$ with 3 twisted rectangles, by arc $b^{\prime}$ with $2 q+1$ crossings as illustrated in Figure28, $q=2,3,4, \cdots$, so that $F^{\prime}(2)$ still has dises $F(2,1)$ and $F(2,2)$ but they are attached by $2 q+1$ twisted rectangles and a similar situation occurs for $B^{\prime}(2)$, then all the previous discussion still holds. Trefoil $b \cup \beta$ is replaced by torus knot $(2,2 q+1)$ and $B^{\prime}(2)$ is a twisted Möbius band. So there are infinitely many constructions possible to obtain a simple knot of genus one with incompressible spanning surfaces of arbitrarily high genus. It is this author's conjecture that the knots are all different.

\section{REFERENCES}

1. Richard F. Gustafson, Construction algorithms for knot spaces which contain incompressible surfaces of arbitrarily high genus, Ph. D. Dissertation, State University of New York at Binghamton, Binghamton, N.Y., 1975.

2. Herbert C. Lyon, Incompressible surfaces in knot spaces, Trans. Amer. Math. Soc., 157 (1971), 53-62.

3. - Simple knots without unique minimal surfaces, Proc. Amer. Math. Soc., 43 (1974), 449-454.

4. Friedhelm Waldhausen, On irreducible 3-manifolds which are sufficiently large, Ann. of Math., (2) 87 (1968), 56-88.

Received October 10, 1979.

State University of New York College at Oneonta

ONEONTA, NY 13820 


\section{PACIFIC JOURNAL OF MATHEMATICS}

\section{EDITORS}

DONALD BABBITT (Managing Editor)

University of California

Los Angeles, California 90024

Hugo RossI

University of Utah

Salt Lake City, UT 84112

C. C. MOORE and ANDREW OGG

University of California

Berkeley, CA 94720

\section{J. DugundJI}

Department of Mathematics University of Southern California Los Angeles, California 90007

R. FinN and J. Milgram Stanford University Stanford, California 94305

\section{ASSOCIATE EDITORS}
R. ARENS
E. F. BECKENBACH
B. H. Neumann
F. WOLF
K. YoshidA

\section{SUPPORTING INSTITUTIONS}

UNIVERSITY OF ARIZONA

UNIVERSITY OF BRITISH COLUMBIA

CALIFORNIA INSTITUTE OF TECHNOLOGY

UNIVERSITY OF CALIFORNIA

MONTANA STATE UNIVERSITY

UNIVERSITY OF NEVADA, RENO

NEW MEXICO STATE UNIVERSITY

OREGON STATE UNIVERSITY
UNIVERSITY OF OREGON

UNIVERSITY OF SOUTHERN CALIFORNIA

STANFORD UNIVERSITY

UNIVERSITY OF HAWAII

UNIVERSITY OF TOKYO

UNIVERSITY OF UTAH

WASHINGTON STATE UNIVERSITY

UNIVERSITY OF WASHINGTON 


\section{Pacific Journal of Mathematics}

\section{Vol. 96, No. $1 \quad$ November, 1981}

Hédi Amara, Groupe des classes et unité fondamentale des extensions quadratiques relatives à un corps quadratique imaginaire principal $\ldots \ldots \ldots 1$

Douglas S. Bridges, On the isolation of zeroes of an analytic function $\ldots \ldots 13$ Andrew J. Casson and John L. Harer, Some homology lens spaces which

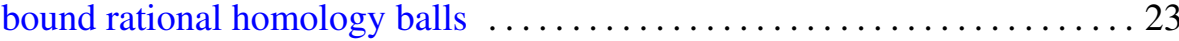

Z. A. Chanturia, On the absolute convergence of Fourier series of the classes $H^{\omega} \cap V[v]$

J.-F. Colombeau and Mário Carvalho Matos, On some spaces of entire functions defined on infinite-dimensional spaces $\ldots \ldots \ldots \ldots \ldots \ldots \ldots 63$

Edwin Duda, Pointwise periodic homeomorphisms on chainable continua . . .77

Richard F. Gustafson, A simple genus one knot with incompressible spanning surfaces of arbitrarily high genus $\ldots \ldots \ldots \ldots \ldots \ldots \ldots \ldots 1$

Fumio Hiai, Masanori Ohya and Makoto Tsukada, Sufficiency, KMS condition and relative entropy in von Neumann algebras

Ted Hurley, Intersections of terms of polycentral series of free groups and free Lie algebras. II .................................. 111

Robert Edward Jamison, II, Partition numbers for trees and ordered sets . . 115 R. D. Ketkar and N. Vanaja, A note on FR-perfect modules ............. 141 Michihiko Kikkawa, On Killing-Ricci forms of Lie triple algebras ....... 153

Jorge Lewowicz, Invariant manifolds for regular points 163

Richard W. Marsh, William H. Mills, Robert L. Ward, Howard Rumsey and Lloyd Richard Welch, Round trinomials .....

Claude Schochet, Topological methods for $C^{*}$-algebras. I. Spectral sequences

Yong Sian So, Polynomial near-fields?

Douglas Wayne Townsend, Imaginary values of meromorphic functions in the disk

Kiyoshi Watanabe, Coverings of a projective algebraic manifold .. 243

Martin Michael Zuckerman, Choosing $l$-element subsets of $n$-element sets 